\title{
A New Methodology for Fiber Optic Strain Gage Measurements and its Characterization
}

\author{
Van Roosbroeck Jan ${ }^{1}$, C. Chojetzki ${ }^{2}$, J. Vlekken ${ }^{1}$, E. Voet ${ }^{1}$, M. Voet ${ }^{1}$ \\ ${ }_{1}^{1}$ Fiber Optic Sensors and Sensing Systems (FOS\&S), Cipalstraat 14, B-2440 Geel, Belgium \\ ${ }^{2}$ FBGS Technologies, Buchaer Straße 6, D-07745 Jena, Germany
}

\begin{abstract}
A new method for mounting fiber optical strain gages to structures will be proposed which is fast, easy and reliable. Mounting of the sensors happens by means of a specially designed mounting tool called a UV sensor pad. It is used in combination with a UV-curable adhesive. The characterization of the performance of these sensors was investigated in an extended research programme and the deduced results will be presented. It will be shown that these sensors exhibit superior characteristics compared to electrical foil gages and hence this is a very promising new measuring technique.
\end{abstract}

\section{INTRODUCTION}

A fibre optic (FO) strain gage consists of an optical fibre with a Fibre Bragg Grating (FBG) which is fixed to a surface by means of an adhesive. This may seem rather straightforward but in practice, this is not at all the case. There are many factors which play a critical role in view of the final sensor performance like e.g. the choice of the adhesive, the fixation length of the fiber and so on. In order to find the best compromise on all these parameters, an extended research project was set up on the account of a major European aircraft manufacturer. The aim was to develop an optical strain gage which can measure strains up to at least $0.5 \%$ (tension and compression) within an operating temperature range of $-45^{\circ} \mathrm{C}$ to $+90{ }^{\circ} \mathrm{C}$. The measurement accuracy should be as high as possibly achievable. Eventually, a final sensor configuration together with the mounting procedure was defined. It is outlined in section 2 . The basic formulas for strain measurement are presented in section 3 and the characterization of these gages with respect to measurement accuracy is outlined in section 4 .

\section{SENSOR DESCRIPTION AND INSTALLATION}

The FBGs used for the current development are the so-called Draw Tower Gratings (DTG) [1]: these gratings are written in the fibre while drawing it and before the fibre coating is being applied. This gives the fibers high strength (breakage at $5 \%$ strain compared to $1 \%$ for standard FBGs) and therefore ensures an excellent fatigue behavior at moderate strain levels. The fiber coating is ORMOCER (Organic Modified Ceramic), which has excellent bonding characteristics with the silica and which has a relatively high Young modulus (about $1 \mathrm{GPa}$ ) assuring a good strain transfer. Therefore, there is no need to remove the coating before fixing the fiber, which largely reduces the risk of fibre breakage during installation.

Fixation of the fiber to a structure is done by means of a specially designed mounting tool called a UV sensor pad, presented in Figure 2. The pad was designed in this way that it can be removed after installation of the sensor. This was deliberately done to avoid excess material around the sensor, which might influence its performance. The sensor thus only consists of the fiber with the FBG and a thin adhesive layer around it. A further advantage of the removable pad is that the sensor can be hermetically sealed by means of commonly used chemical sealants and / or mechanical protection layers.

The fiber with FBG to be used with the pad is specially configured so that it can be directly mounted in the sensor pad. It is schematically depicted in Figure 1. The fiber is protected inside a $900 \mu \mathrm{m}$ diameter FEPtubing over its entire length, except the area around the FBG (see label 5 in the figure). Here, the fiber is free of tubing over a length of $28 \mathrm{~mm}$ with the FBG located in the centre. One end is connected to a FC/APC connector and the other end is angle cleaved in order to avoid reflections. 


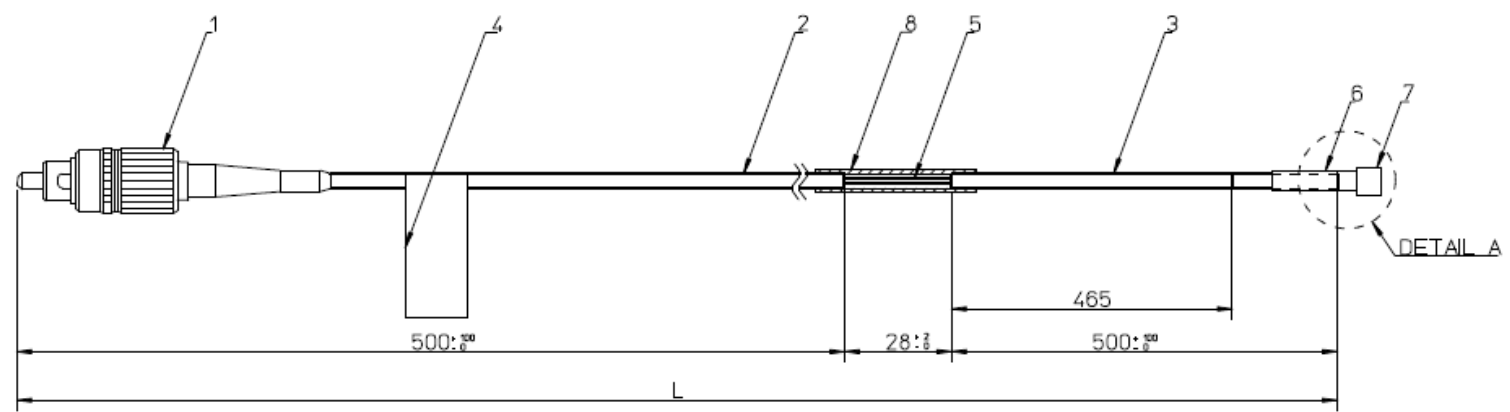

Figure 1: Schematic drawing of the SG-01 Strain Gage, to be used with the UV sensor pad.

The sensor pad is direcly clipped on the $900 \mu \mathrm{m}$ tubing left and right from the free fiber zone (see Figure 2). This way, the free fiber with FBG is located at the down side of the sensor pad. The adhesive will be put on this side of the pad so that the fiber and adhesive can make direct contact with the surface to which they will be attached.
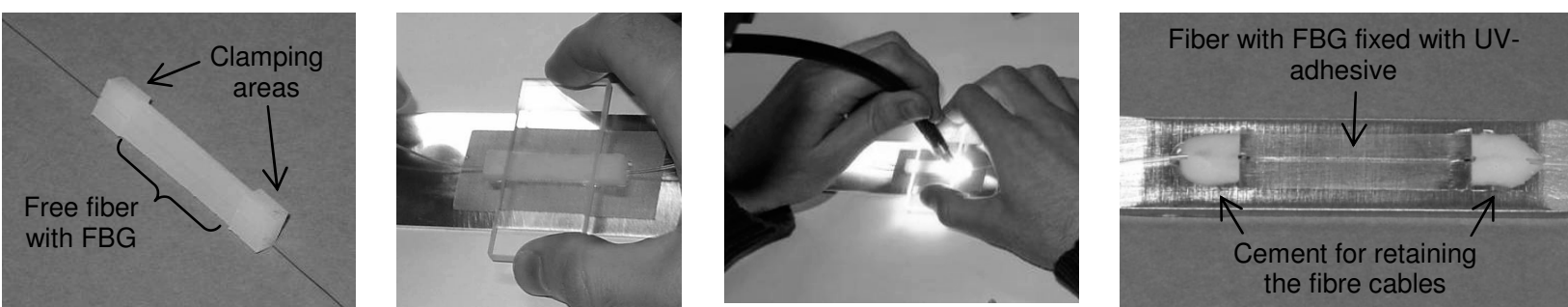

Figure 2: The different stages during installation: (1) the UV sensor pad holding the sensing fiber, (2) manual pressurization of the pad, (3) UV-curing and (4) the installed FO strain gage.

Installation of the sensor pad to the structure to be measured happens by means of a UV-curable adhesive. This type of adhesive was found to give the best compromise between curing time, operating temperature range and practicality. The area of the glue zone on the sensor pad is $30 \times 8 \mathrm{~mm}$. Installation happens with a stringent fixation procedure, illustrated in Figure 2. It can be summarized as follows: (1) prepare the surface (similar as for electrical gages); (2) mount the sensor pad on the sensing fibre (photo 1); (3) apply the adhesive and sensor pad to the structure; (4) pressurize the pad manually by means of a special glass piece in order to keep the fiber under slight tension and to control the adhesive thickness (photo 2); (5) pre-cure with UV-light for 30 seconds (photo 3); (6) remove the glass piece and sensor pad; (7) post-cure with UV-light for typically 5 to 10 minutes; (8) secure the cables with cement (photo 4) and (9) optionally apply chemical sealants and / or mechanical protection layers.

To achieve optimal performance, the sensor pad was developed with respect to: (1) easy fixation and removal of the pad to the fiber cable; (2) semi-transparency for UV-light; (3) slight fibre pre-straining when being pressed; (4) sufficient flexibility so that it bends around the fiber when being pressed and (5) no bonding with the adhesive so that it can be removed after curing.

\section{STRAIN MEASUREMENT BASICS}

The strain $\varepsilon$ measured by the FO gage can be derived from the differential formula [2]:

$$
\frac{d \lambda}{\lambda}=S_{\varepsilon} \cdot d \varepsilon+\Sigma . d T
$$

where $\lambda$ is the wavelength, $S_{\varepsilon}$ the gage factor $\left[S_{\varepsilon}=(1-P)\right.$ with $P$ the strain-optic coefficient], $T$ the temperature and $\Sigma$ the temperature sensitivity as a result of the intrinsic temperature sensitivity of the FBG and of the thermal expansion of the material to which the gage is attached. Integrating both sides of this formula yields:

$$
\ln \frac{\lambda}{\lambda_{0}}=S_{\varepsilon} .\left(\varepsilon-\varepsilon_{0}\right)+\Sigma .\left(T-T_{0}\right)=S_{\varepsilon} . \Delta \varepsilon+\Sigma . \Delta T
$$

where $\lambda_{0}$ indicates the reference Bragg wavelength at reference strain $\varepsilon_{0}$ and at reference temperature $T_{0}$. It should be noted that equation (1) is often approximated without performing the above integration procedure ( $d$ is replaced by $\Delta$ and $\lambda$ by $\lambda_{0}$ ). However, this introduces errors which increase for increasing 
strain. The induced error is of the order of $0.2 \%$ relative strain error for $0.5 \%$ strain. Equation (2) is more appropriate in this context and thus will be used henceforth. Equation (2) can be rewritten as:

$$
\ln \frac{\lambda}{\lambda_{0}}=S_{\varepsilon} \cdot\left(\Delta \varepsilon+\frac{\Sigma}{S_{\varepsilon}} \Delta T\right)
$$

where the term $\left(\Sigma / S_{\varepsilon}\right) \cdot \Delta T$ is usually referred to as the thermal output. This term represents the apparent strain change due to temperature changes caused by the intrinsic temperature sensitivity of the gage and by the thermal expansion of the material to which the gage is attached. In case of pure strain measurements, the thermal output should be accounted for. For electrical gages, similar effects occur and the thermal output needs to be accounted for as well. A possible way to eliminate the thermal output is to use a second gage which is mounted on a compensating plate, see Figure 3. This plate is made of the same material as that to which the first gage is attached and has a cantilever shape in order to avoid strain transfer from the structure to the gage on the plate $(\Delta \varepsilon=0)$. Hence, the gage on the compensating plate (subscript $T$ ) only produces a thermal output and this can then be used to

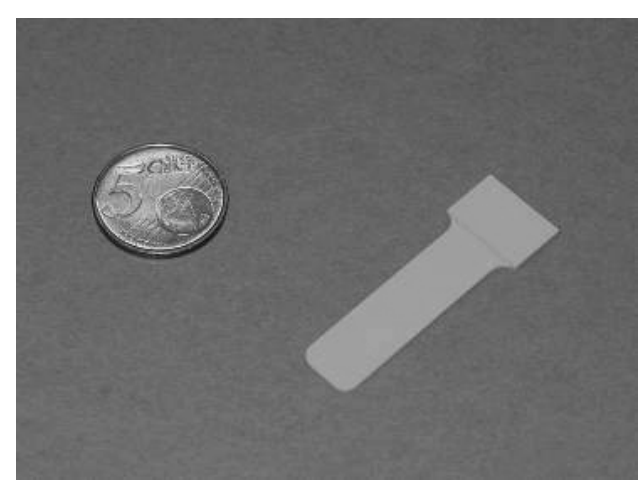

Figure 3: Cantilever shaped compensating plate. eliminate the thermal output from equation (3):

$$
\Delta \varepsilon=\frac{1}{S_{\varepsilon}}\left[\ln \frac{\lambda}{\lambda_{0}}-\ln \frac{\lambda_{T}}{\lambda_{T 0}}\right]
$$

\section{ACCURACY ESTIMATION}

\subsection{Statistical error on the gage factor}

The gage factor $S_{\varepsilon}$ of a strain gage can only be measured when the gage is already installed and since the gages cannot be re-used, it is impossible to do a calibration of each sensor individually. Therefore, one should derive the gage factor statistically from a number of samples taken at random from a production batch. For this reason, the measurement accuracy strongly depends on the reproducibility of the gage factor. In order to determine the statistical error on the gage factor, strain calibrations were executed on 4 gages from the same batch. They were mounted on a 4-point bending beam apparatus which was built according to the standards [3, 4]. The strain was varied from $-1000 \mu \varepsilon$ to $+1000 \mu \varepsilon$ in steps of $200 \mu \varepsilon$. Equation (2) was used for deriving the gage factor $S_{\varepsilon}$ under the assumption that the temperature remained stable $(\Delta T=0)$. The mean value for the gage factor of the 4 gages was found to be 7.886. $10^{-7} \mu \varepsilon^{-1}$. The relative variations of the individual gage factors with respect to the mean value were of the order of $0.1 \%$ and hence the reproducibility is quite satisfactory. The statistical error was estimated from the standard deviation using standard statistical methods [4] and was found to be 2.1.10-9 $\mu \varepsilon^{-1}$. Hence, the relative statistical error is of the order of $<0.5 \%$.

\subsection{Error due to the temperature dependence of the gage factor}

The gage factor is usually derived at room temperature but is in general temperature dependent. In a first approximation, it is assumed that the gage factor varies linearly with temperature, according to the formula $[3,4]$ :

$$
S_{\varepsilon}(T)=S_{\varepsilon}\left(T_{0}\right) \cdot\left[1+\alpha \cdot\left(T-T_{0}\right)\right]
$$

where $S_{\varepsilon}(T)$ is the temperature dependent gage factor, $T_{0}$ the calibration temperature and $\alpha$ the relative temperature dependence of the gage factor. The latter was deduced experimentaly by measuring the temperature induced wavelength shifts from pairs of FO strain gages mounted on sample plates of the same material (same $\Sigma$ ) but mounted with different initial strains. The sample plates with the gages were kept at various temperatures $T$ and their wavelengths $\lambda_{1}$ and $\lambda_{2}$ were recorded. The following set of equations holds for each pair of FBGs:

$$
\left\{\begin{array}{c}
\ln \lambda_{1}=S_{\varepsilon}(T) \cdot \varepsilon+\Sigma \cdot T+C_{1} \\
\ln \lambda_{2}=S_{\varepsilon}(T) \cdot\left(\varepsilon+\Delta \varepsilon_{0}\right)+\Sigma \cdot T+C_{2}
\end{array}\right.
$$


with $\Delta \varepsilon_{0}$ the difference in pre-strain between the two gages and $C_{1}$ and $C_{2}$ temperature independent quantities taken over from equation (2). Taking the difference between both equations and inserting equation (5) yields:

$$
\ln \frac{\lambda_{2}}{\lambda_{1}}=\alpha \cdot S_{\varepsilon}\left(T_{0}\right) \cdot \Delta \varepsilon_{0} \cdot\left(T-T_{0}\right)+C_{3}
$$

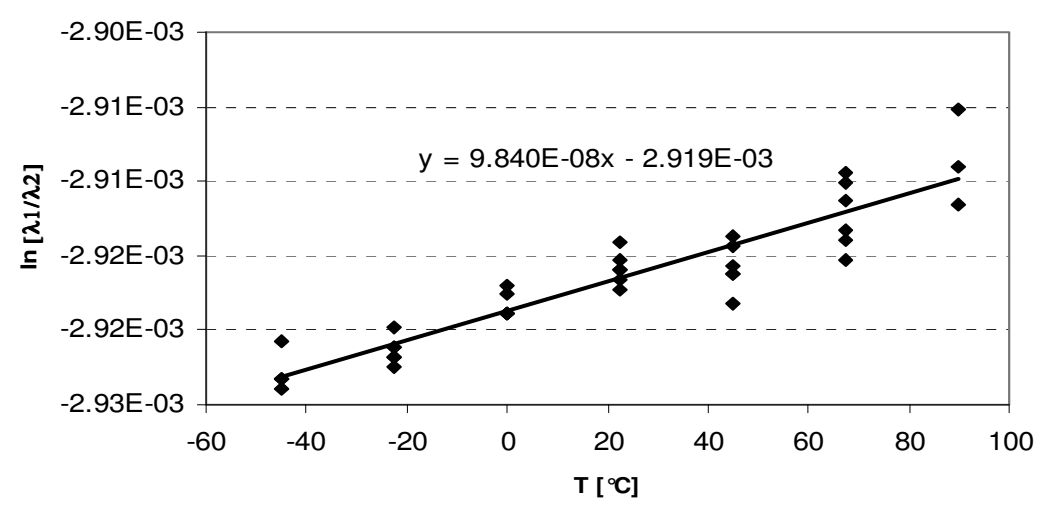

Figure 4: Plot of In $\left(\lambda_{2} / \lambda_{1}\right)$ versus T for a pair of FBGs mounted on a steel sample plate with $\Delta \varepsilon_{0}=408 \mu \varepsilon$.

with $C_{3}$ a temperature independent term. The plot of $\ln \left(\lambda_{1} / \lambda_{2}\right)$ versus $T$ thus yields a linear curve with slope $\alpha . S_{\varepsilon} \cdot \Delta \varepsilon_{0}$, see Figure 4 , and hence $\alpha$ can be deduced provided that the difference in pre-strain $\Delta \varepsilon_{0}$ is known. This procedure was applied on different metals: aluminum, steel and stainless steel. The mean value derived for $\alpha$ was $(2.7 \pm$ 0.4). $10^{-4}{ }^{\circ} \mathrm{C}^{-1}$.

This value is in good accordance with the literature value of $3.02 .10^{-4}$ ${ }^{\circ} \mathrm{C}^{-1}$ from [5], taken into account the nominal wavelength of the used gage $(850 \mathrm{~nm})$. When the gage factor is specified at room temperature $\left(20^{\circ} \mathrm{C}\right)$, the maximum possible temperature shift in the range of $-45^{\circ} \mathrm{C}$ to $+90^{\circ} \mathrm{C}$ would be $70^{\circ} \mathrm{C}$. This corresponds to a relative gage factor variation of $2.710^{-4} \times 70=1.9 \%$.

\subsection{Error due to transverse sensitivity}

The transverse sensitivity is the ratio of the gage factor of a strain gage mounted perpendicular to an unidirectional strain field (transverse gage) to the gage factor of a similar gage mounted parallel to the same strain field (longitudinal gage) [3, 4]. The transverse sensitivity of the optical gages was determined by mounting 4 optical gages perpendicular to the unidirectional strain field of a test rig constructed according to reference $[3,4]$. The unidirectional strain was varied from 0 to $1200 \mu \varepsilon$. The mean and maximum strain as indicated by the gages was found to be $2.5 \mu \varepsilon$ and $3.3 \mu \varepsilon$ respectively. This means that the transverse sensitivity is of the order of $2 \cdot 1 \cdot 10^{-3}$. The observed effect was however most likely an artifact from the used test rig because of its imperfectness. The true transverse sensitivity therefore is probably even lower and the above value should be considered as an upper limit.

\subsection{Error due to fatigue effects during mechanical loading}

Fatigue experiments were carried out for FO gages fixed to different sample plates in order to investigate the bonding characteristics of the gages to different materials. The evaluated sample materials were aluminum 2024, steel, stainless steel (inox), inconel, titanium TA6V, carbon composite and UD glass composite (Aerospace FP525). The metallic samples were manufactured according to the ASTM-norm [6]. The composite samples had a plain rectangular shape. The samples were clamped in an Instron FastTrack 8801 strain bench and the strain was varied according to a sine-wave pattern. All samples except the FP525 were tested in tension between 0 and $1000 \mu \varepsilon$. The FP525 was strained both in tension and compression between $-0.24 \%$ and $+0.24 \%$. The number of load cycles was in all cases at least 2 million, except for the titanium samples which were only tested up to 300.000 cycles because of early material failure. The frequency of cycling was $15 \mathrm{~Hz}$ for the metallic samples and $5 \mathrm{~Hz}$ for the composite samples (reduced frequency due to increased heat generation of the composite samples). At regular time intervals (every 75.000 cycles), a quasi-static calibration was performed in order to monitor possible drifts in the gage factor and of the zero strain value. A reference measurement was performed with an electrical extensometer. In addition, a temperature correction procedure was performed in order to compensate for the variations of the lab temperature. Figure 5 shows the relative variations of the gage factor and the absolute zero shifts after the temperature correction for all samples. The slope variations of the FP525 sample could not be derived since the FBG-peak exhibited deformations during tension of the sample which could be traced back to micro-cracks at the surface of the sample. Both slope and offset show some initial setting. This is most likely not related to the FO strain gage and is definitely not related to fatigue effects. Based on the graphs from Figure 5, upper limits of the fatigue shifts could be estimated based on the $95 \%$ confidence interval of a linear fit procedure. If the deduced fatigue shift is smaller than 
the $95 \%$ confidence interval, the shift can be considered as being insignificant. The observed slope drifts were all found to be insignificant for all of the tested samples. The zero shifts were also found to be insignificant for all metallic samples. For the composite materials, an upper limit of the zero shift of $6 \mu \varepsilon /$ $10^{6}$ cycles could be estimated based on the $95 \%$ confidence intervals.
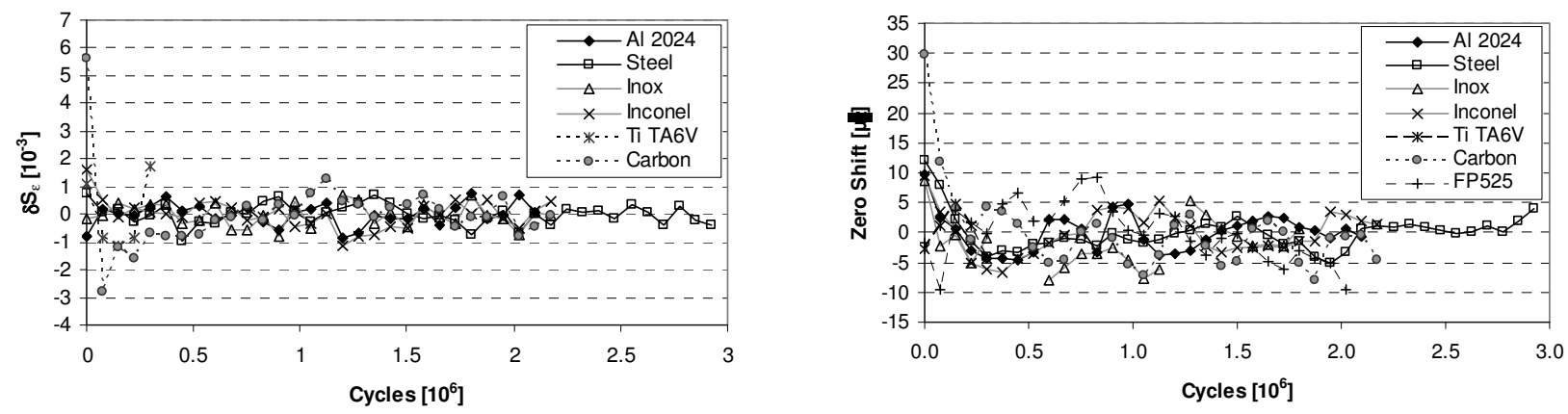

Figure 5: Relative variations of the gage factor (left) and absolute zero shifts as a function of the number of fatigue cycles for various sample materials.

Altogether, it can be concluded that the observed fatigue effects are negligible or even non-existing. In particular, the upper limit of $6 \mu \varepsilon / 10^{6}$ cycles for the FP525 composite material shows the superiority of this type of FO gages since this is an exceptional performance as compared to their electrical strain gage counterparts.

\subsection{Error due to thermal cycling}

In order to investigate the effects of thermal cycling, temperature calibrations on the fixed strain gages were performed. The gages were mounted on metallic samples that were placed in a Metrology Well calibrator 9170 from Fluke. Five calibration cycles from $-45^{\circ} \mathrm{C}$ to $90^{\circ} \mathrm{C}$ were executed. The resulting calibration is shown in Figure 6.
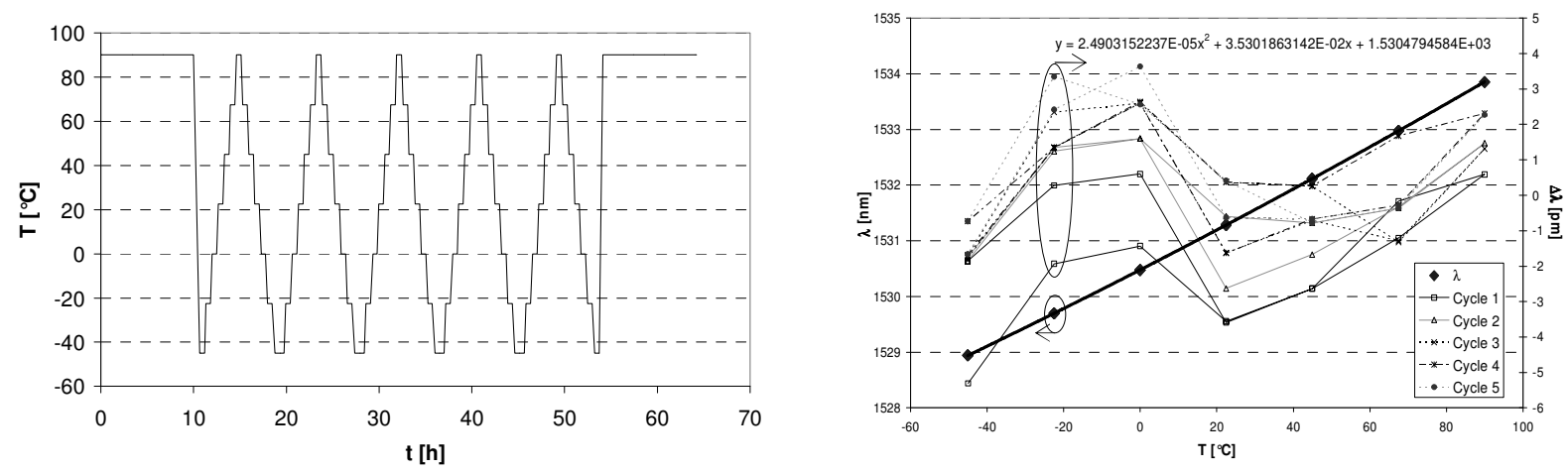

Figure 6: Used temperature profile (left) and corresponding calibration data ( $\lambda$ versus $T)$. The differences of the data with the quadratic fit $(\Delta \lambda)$ for the individual cycles are also shown.

The differences of the data points with the quadratic fit curve are also shown in the figure for all calibration cycles individually. As can be seen, these differences are reproducible down to roughly $\pm 6 \mathrm{pm}$ or $5 \mu \varepsilon$. The error due to temperature cycling therefore is of the order of $5 \mu \varepsilon$.

\subsection{Error due to high temperature drifts}

When the FO gages are kept for extended periods of time at elevated temperatures, it was observed that the wavelength slightly drops as a function of time, see Figure 7 . This creep effect could be traced back to a combination of the relatively short fibre fixation length, the adhesive becoming weaker at elevated temperatures and the increased thermal expansion of the substrate material. The creep limits the operating temperature of the $\mathrm{FO}$ gages. At $90^{\circ} \mathrm{C}$, the creep effect causes the wavelength to drop a few microstrain per hour. An upper limit of $5 \mu \varepsilon / \mathrm{h}$ could be indicated, including also a safety margin. At $70^{\circ} \mathrm{C}$, an upper limit of $0.026 \mu \varepsilon / \mathrm{h}$ could be determined. 

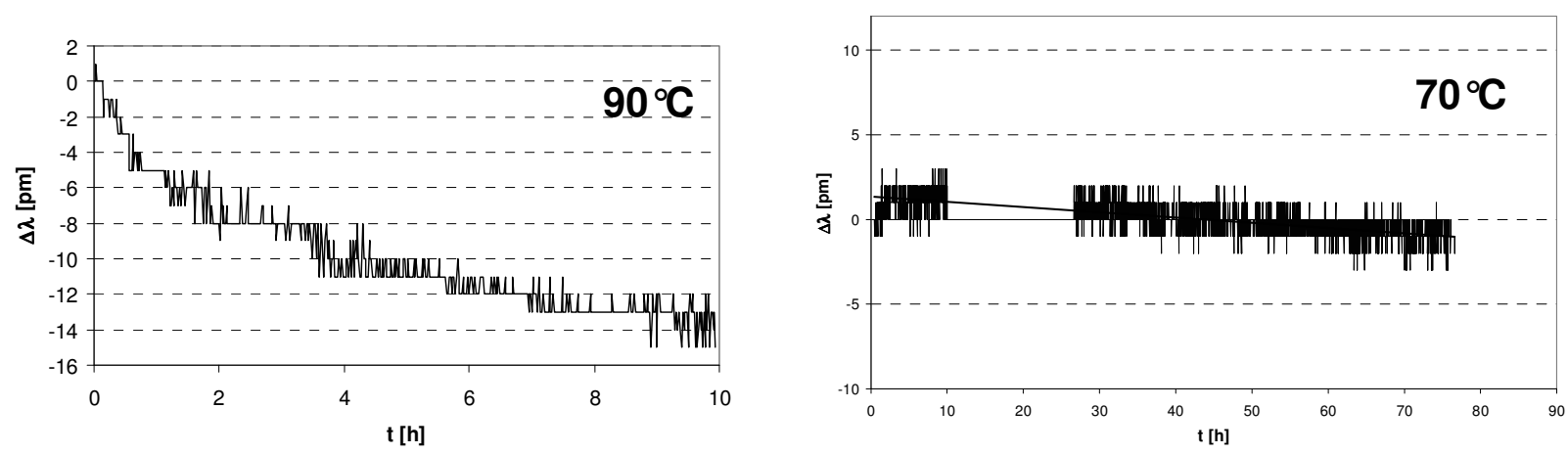

Figure 7: Wavelength stability of the FO gages during extended periods of time at $90^{\circ} \mathrm{C}$ (left) and $70^{\circ} \mathrm{C}$.

\subsection{Error due to thermal output}

Equation (4) is the general formula to be used in case of temperature compensation with a compensating plate. However, it is only valid under the assumption that both gages (1) are mounted on the same material, (2) have the same gage factor and (3) experience the same temperature. The first condition can easily be met. Seen from section 4.1 , the second condition is also valid. The third condition will be more difficult to realize in general. The measurement error will depend on the experimental conditions and therefore cannot be formulated quantitatively.

\section{CONCLUSIONS}

A new methodology for fiber optic strain gage measurements has been presented and the performance of the gages has been characterized in an extended research programme. The most prominent error sources were identified, measured and are listed in Table 1. Most of these properties are well known error sources for electrical gages. It can be concluded that the here presented FO gages exhibit superior characteristics compared to the electrical foil gages and hence this is a very promising new measuring technique.

\begin{tabular}{|c|l|c|}
\hline Parameter & \multicolumn{1}{|c|}{ Error Source } & Error \\
\hline \multirow{4}{*}{$S_{\varepsilon}$} & Statistical error & $0.5 \%$ \\
\cline { 2 - 3 } & Temperature coefficient & $2.710^{-4} \mathrm{C}^{-1}$ \\
\cline { 2 - 3 } & Transverse sensitivity & $<2.1 .10^{-3}$ \\
\cline { 2 - 3 } & Relative slope shift due to fatigue cycling & Insignificant \\
\hline \multirow{4}{*}{$\lambda$} & Zero shift due to fatigue cycling & $<6 \mu \varepsilon / 10^{6} \mathrm{cycles}$ \\
\cline { 2 - 3 } & Thermal cycling & $<5 \mu \varepsilon$ \\
\cline { 2 - 3 } & Thermal drifts at elevated temperature & $\begin{array}{c}\leq-0.026 \mu \varepsilon / \mathrm{h} @ 70^{\circ} \mathrm{C} \\
\leq-5 \mu \varepsilon / \mathrm{h} @ 90^{\circ} \mathrm{C}\end{array}$ \\
\cline { 2 - 3 } & Thermal output & Depends on \\
& & measurement conditions \\
\hline
\end{tabular}

Table 1: Overview of the most prominent error sources for the here proposed FO strain gages.

This work has been developed in the framework of a joint collaboration between the Belgian Science Policy and the Federal Public Service of Economy, SMEs, Independent Professions and Energy of Belgium.

[1] www.fbgs-technologies.com

[2] Butter, Hocker, "Fiber Optics Strain Gauge", Applied Optics 17, 18 (1978)

[3] ASTM-norm E 251-92, "Standard Test Methods for Performance Characteristics of Metallic Bonded Resistance Strain Gages", Annual Book of ASTM Standards Vol. 03.01. (2003)

[4] "Experimentelle Strukturanalyse Dehnungsmessstreifen mit metalilischem Messgitter Kenngrößen und Prüfbedingungen", VDI/VDE/GESA-Richtlinien 2635 (Germany, 2005)

[5] M.J. O'Dwyer et al., "Thermal Dependence of the Strain Response of Optical FBGs", Measurement Science and Technology 15 (2004) 1607-1623.

[6] ASTM-norm E 8M-04, "Test Methods for Tension Testing of Metallic Materials", Annual Book of ASTM Standards Vol. 03.01. 